(C) [2010] IEEE. Reprinted, with permission, from [Valérie Gay, Peter Leijdekkers and Edward Barin, Feasibility Trial of a Novel Mobile Cardiac Rehabilitation Application, e-Health Networking Applications and Services (Healthcom), 2010 12th IEEE International Conference on, 1-3 July 2010]. This material is posted here with permission of the IEEE. Such permission of the IEEE does not in any way imply IEEE endorsement of any of the University of Technology, Sydney's products or services. Internal or personal use of this material is permitted. However, permission to reprint/republish this material for advertising or promotional purposes or for creating new collective works for resale or redistribution must be obtained from the IEEE by writing to pubs-permissions@ieee.org. By choosing to view this document, you agree to all provisions of the copyright laws protecting it 


\section{Feasibility Trial of a Novel Mobile Cardiac Rehabilitation Application}

\author{
Valérie Gay and Peter Leijdekkers \\ Faculty of Engineering and IT \\ University of Technology, Sydney \\ Australia \\ \{Valerie.Gay, Peter.Leijdekkers\}@uts.edu.au
}

\author{
Edward Barin \\ Cardiology Department \\ Royal North Shore Hospital, \\ Sydney, Australia \\ Edward.Barin@gmail.com
}

\begin{abstract}
A trial with a cardiac rehabilitation centre is in progress where we test a novel cardiac rehab application using a standard mobile phone and wireless sensors. The goal is to obtain insight how remote monitoring compares to conventional rehabilitation methods in terms of adherence to cardiac rehabilitation programmes. In this trial we seek feedback from patients and health professionals regarding usability and practicability of the software and hardware used and we investigate whether the use of the novel mobile rehabilitation application provides clinically meaningful reassurance to patients during their cardiac rehabilitation. The trial also investigates whether physical and psychological measures improve using the system. This paper describes the mobile cardiac rehabilitation application as well as the setup of the trial.
\end{abstract}

Keywords_- Remote health monitoring, cardiac rehabilitation

\section{INTRODUCTION}

Cardiac rehabilitation programmes aim at maximizing physical, psychological and social functioning of a patient following a myocardial infarction or coronary bypass surgery [1]. The benefits of rehabilitation have been demonstrated by several research groups and it can reduce mortality, lower blood pressure, improve lipids and stress levels [2, 3, 4]. However, despite strong evidence that cardiac rehabilitation reduces disability and prolongs life, fewer than one in five people in the USA receive rehabilitation services after a heart attack or coronary bypass surgery [5]. This is sometimes due to access issues (geography, lack of resources or ill-health) but often due to lack of motivation since it is hard to make lifestyle changes without any external help. Another issue is that many patients do not complete the rehabilitation programme and pull out before the desired health style changes have been achieved.

For people having access issues, home based cardiac rehabilitation has been widely noted as a viable alternative to rehabilitation programmes conducted at a hospital [6]. A study [2] in Canada and Australia found that remote monitoring could help patients that do not benefit from in-house rehabilitation services. It also showed that the use of remote monitoring of physiological data helps reduce heart failure admissions to hospitals and lowers the mortality rate by nearly twenty per cent for chronic heart failure patients. Their study highlighted that remote monitoring has the potential to significantly improve clinical outcomes (mortality, morbidity and quality indicators).
To improve long term clinical outcomes, patients need to make lifestyle changes and this is not something that can be achieved in a few weeks. The patients need to integrate exercises in their daily life; they need to understand the cause and effect of medication and activities on their health. It is important for the patients to keep track of their health and be reminded and motivated to exercise.

Substantial research has been done on mobile monitoring systems, which typically incorporate wearable or implanted sensors and run software on a mobile device to perform a set of functions around one clinical application. CardioMobile [7] defines a system for real-time remote monitoring of ECG, heart rate, location and speed. The CSIRO e-Health project [8] focuses on the assessment of cardiac rehabilitation patients in a community care model. The main differences between our mobile rehabilitation application and existing ones are the following: our application combines activity and biosignal monitoring (e.g. exercise, ECG, weight, blood pressure, glucose). It provides immediate local feedback to the patient without the intervention of a health professional. It can also be used for real-time remote monitoring of a patient using 3G technology. Health professionals can use a standard web browser to access their patients' data and carry out remote monitoring and reporting. Our rehabilitation application is integrated into a platform that offers useful functionalities such as arrhythmia detection and emergency facilities such as automatic emergency calls in case of detection of a fall or of a life-threatening arrhythmia.

In this paper we discuss a trial that is in progress at a cardiac rehabilitation centre in Sydney (Australia) using the mobile cardiac rehabilitation application. The aim is to investigate whether remote monitoring of cardiac patients can improve long term clinical outcomes. We want to study how remote monitoring compares to conventional rehabilitation methods in terms of helping patients monitoring their vital signs and making lifestyle changes. In this trial we seek feedback from patients and health professionals regarding usability and practicability of the software and hardware used and we investigate whether the use of the novel application provides clinically meaningful reassurance to patients during their cardiac rehabilitation.

The participants in the trial have all recently experienced an acute cardiovascular event and joined the rehabilitation programme at the cardiac rehabilitation centre. We followed the CONSORT (CONsolidated Standards Of Reporting Trials) 
recommendations [9] to set up a so-called parallel-group randomized trial where one group uses the mobile rehab application consisting of mobile phone and sensors. A second group uses the mobile rehab application without sensors and has to enter all data manually. The third group does not use the mobile phone application and keep track of their progress and measurements on paper. To ensure that the results are not influenced by paying extra attention to patients involved in the trial we have a fourth group that is not involved in the trial at all and results will be compared against this group.

This paper describes the mobile rehabilitation application in section 2 and how it potentially can improve rehab compliance. Section 3 details the trial setup and section 4 concludes with the next steps to be taken.

\section{A NOVEL REMOTE REHABILITATION SYSTEM}

A novel rehabilitation system has been developed at the University of Technology, Sydney to help patients and rehabilitation professionals, dealing with adherence to a rehabilitation program.

The rehabilitation module is integrated in the existing Personal Health Monitor (PHM) application [10, 11] which offers real-time personalised vital signs monitoring using a standard mobile phone and wireless sensors (Fig. 1).

The patient is monitored using a mobile phone and one or more wireless sensors. The phone reminds the patient when measurements or a workout session is due. Patients can keep track of their blood pressure, weight history and can monitor the progress of their rehabilitation program both on the mobile phone and web based interface on their home computer.

The health professional can remotely manage and check the information about their patients using the web based system.

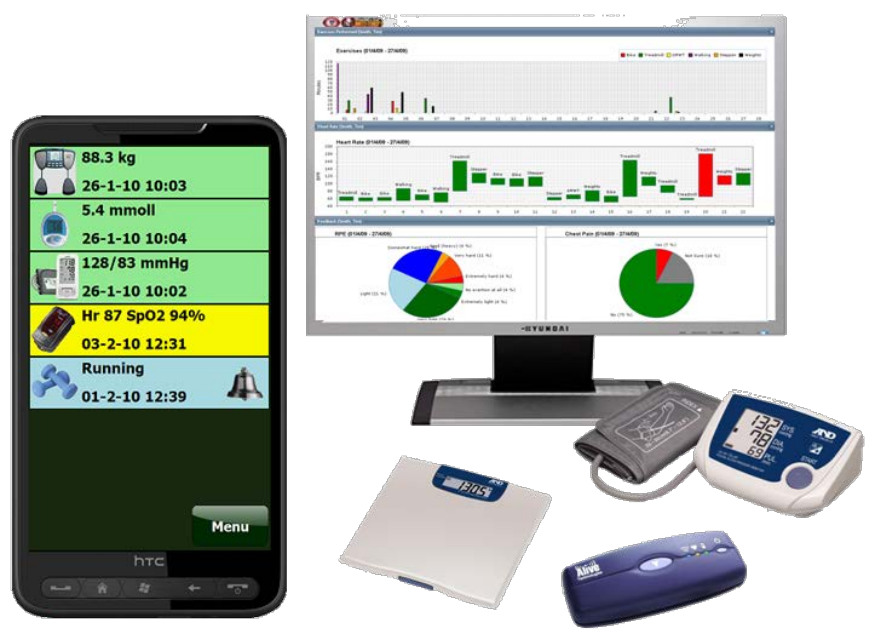

Figure 1. Personal Health Monitor Rehabilitation System

They can remotely personalise and adapt patient reminders and thresholds (e.g. max heart rate set to $120 \mathrm{bpm}$ ) and use the collected information to generate reports.

Changes made by the health professional and data collected on the mobile phone are synchronised periodically, giving both the patient and the health professional access to the latest and most accurate data.

The integrated approach enables the collection of complementary information while doing their exercises. That is, the vital sign measurements (e.g. blood pressure, heart rate, blood glucose) can complement the self-reported effects.

The PHM system is a web-based system accessible by different stakeholders. Fig. 2 shows a high level view of the several stakeholders that interact with the system.

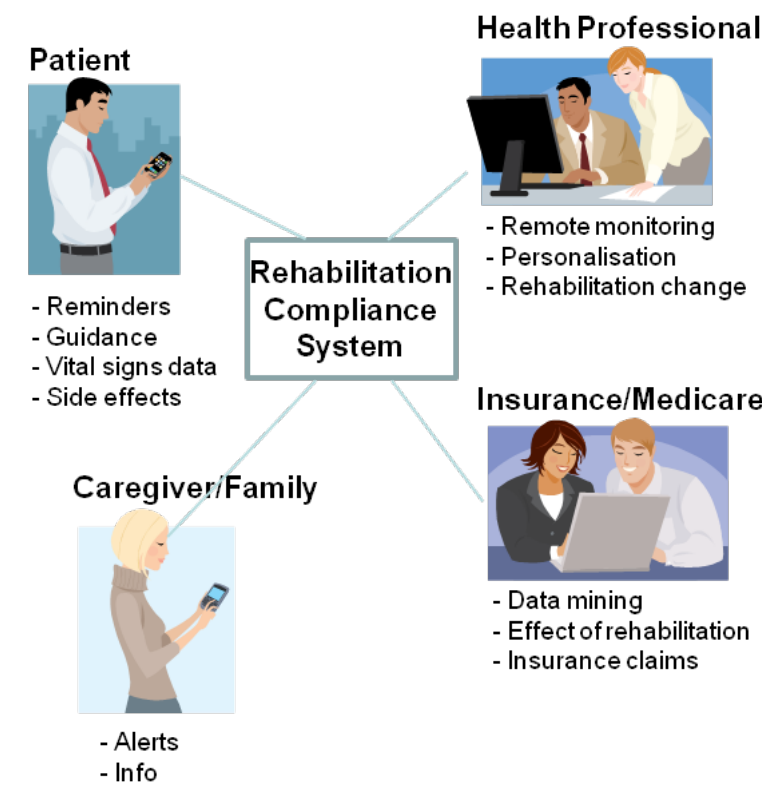

Figure 2. Stakeholders involved in the PHM Rehabilitation System

The Health Professional (personal trainers, rehabilitation specialists) can monitor their patients' compliance to their rehabilitation programme. Higher compliance rate means that health professionals can make better informed decisions on the effects of the rehabilitation programme. They can monitor the progress and adherence together with vital signs information and the patient's subjective feedback.

Family members and caregivers may find it difficult to monitor the patient's adherence to a rehabilitation program. Family members of an elderly patient may want to know that the exercises have been done and be able to personally remind the elderly person of doing their exercises. With the patient's permission, they can subscribe to a service to receive alerts on their mobile phone when a rehabilitation session is due, or have access to the rehabilitation history on the website. If the patient is not compliant for a period of time, an email or SMS can notify caregivers of the problem.

Public and private health insurance companies can connect to the system and request anonymous rehabilitation compliance information. This information can help them in developing data mining techniques to assess the effect of rehabilitation programmes. Special rehabilitation programmes 
can be introduced to target certain age groups in order to improve the outcomes.

Patients are the main beneficiary of the system since it helps them overcome the reasons why they do not comply with their rehabilitation programme. In the introduction, we identified several issues why patients do not adhere to their rehabilitation programme. The rehabilitation module developed tries to address these issues.

Issue 1: 'lack of motivation, unwilling to make lifestyle changes'. This is a probably the most difficult issue to solve, even using technology. However, if the mobile phone (which most people use every day) keeps reminding the person to do their exercises it might have the effect that they will actually do the exercises (Fig. 3). Not doing the exercises will be recorded and after a period of time a health professional or family member might perform peer pressure on the person to do their exercises. Our technology provides the means to gather the data whether the person adheres to the rehabilitation programme and appropriate action can be taken.
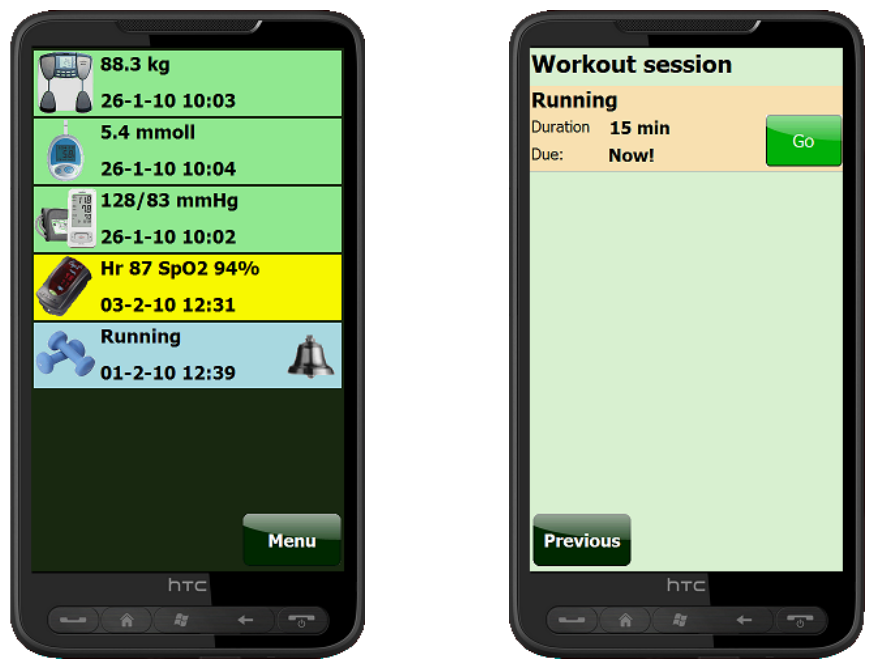

Figure 3: Rehabilitation reminders

Issue 2: 'Limited access to rehabilitation programs due to geography, lack of resources or ill health'. The rehabilitation system consists of a mobile phone and one or more sensors and can be used anywhere (indoors, outdoors, at the gym). The system can be remotely configured by a rehabilitation specialist and there is no need to see a specialist in person to make changes to the rehabilitation program. This makes it very suitable for patients living in remote areas but also to others not having easy access to a specialist. The rehabilitation system can monitor one or more vital signs for those patients that need monitoring while performing their exercises. Blood pressure readings can be taken before, during and after an exercise session to monitor any adverse effects (Fig. 4). If dramatic changes occur the application will warn the person to either stop with the exercises or seek medical attention. For cardiac patients an ECG monitor can be used to monitor heart rate threshold levels as well as arrhythmia monitoring. Automatic ECG recordings are made in case of abnormalities.
This allows a rehab specialist to remotely monitor the effect of the exercises on the patient.
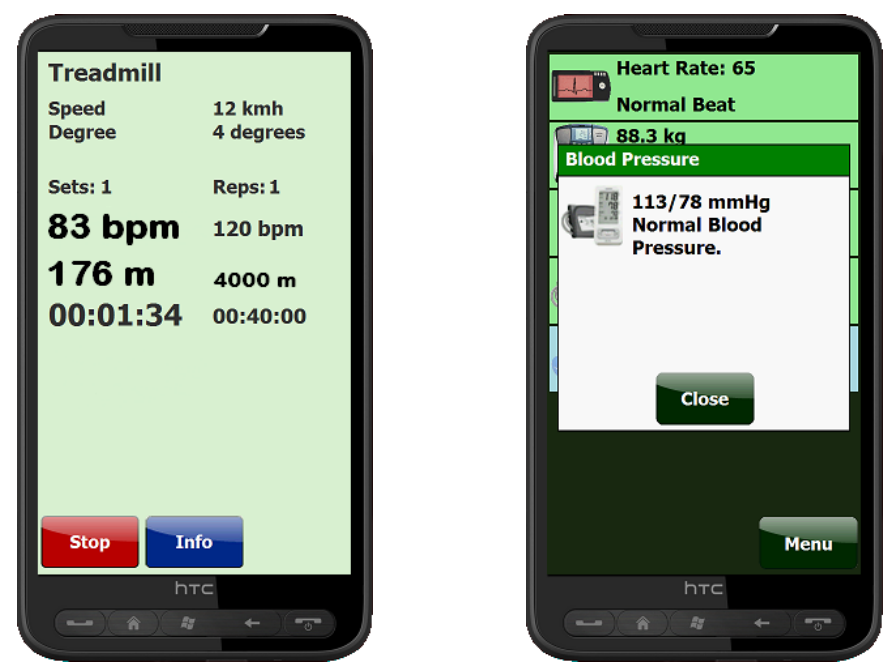

Figure 4: Using sensors to monitor vital signs during exercise

The application will guide the user through the exercises that need to be performed. At any time the user can obtain detailed information about the exercise (Fig 5). The user can choose between images and text describing the exercise or play a audio/video file demonstrating the exercise.
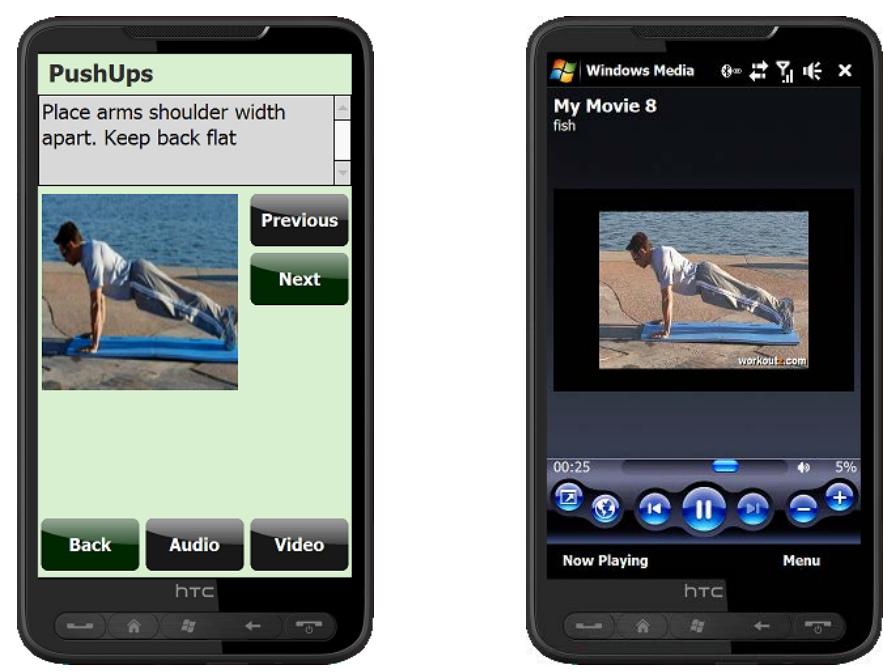

Figure 5: Detailed exercise instructions

Cardiac patients often experience the anxiety of not wanting to push too hard while doing their exercises. However feedback received from rehabilitation specialists suggest that most patients underperform. Patients do not dare pushing themselves while doing the exercises. The mobile rehabilitation application addresses this issue by giving realtime feedback to the users when they do their exercises. Using an ECG heart monitor the application can monitor the heart rate. This gives a fair indication whether the patient is pushing hard enough. In addition the system collects the patient's feedback using the Borg Rating of Perceived Exertion (RPE) scale. This gives a subjective view how the user perceived the difficulty of the exercise. 

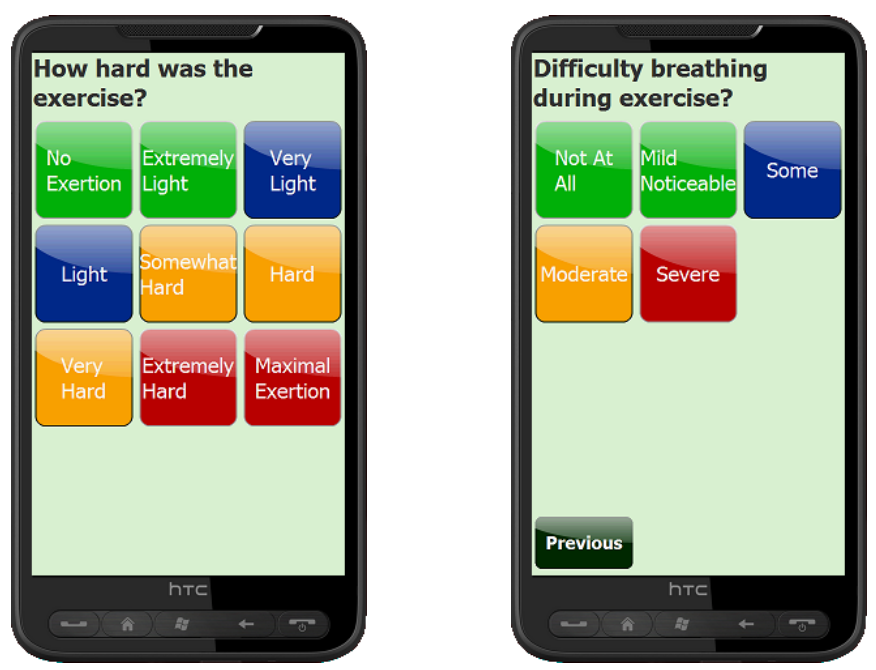

Figure 6: Feedback reported to health professionals

Issue 3: 'Provide strategies to help patients maintain longterm compliance'. The rehabilitation application can be used standalone on the mobile phone where patients keep track of their progress. The data can also be uploaded to the web site (www.PersonalHeartMonitor.net) where health professionals can remotely monitor the patient's progress. New thresholds and exercise information can be set remotely by the rehabilitation specialist and uploaded to the mobile phone using 3G.

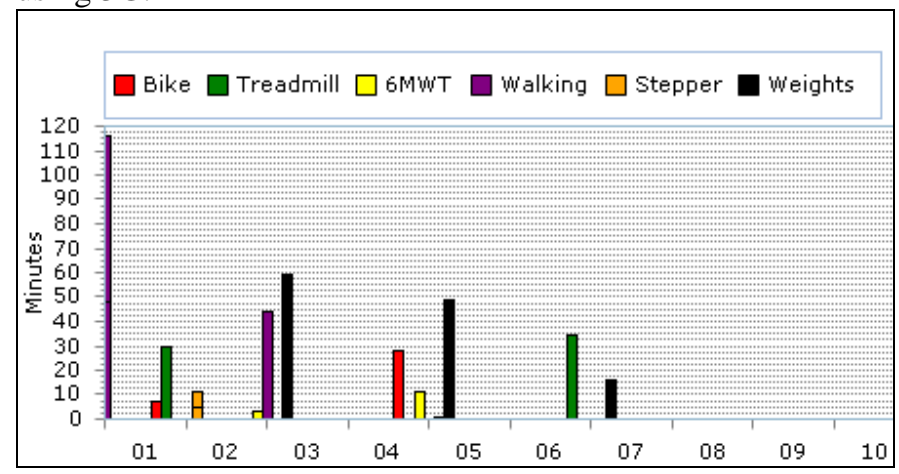

Figure 7: Exercises performed over a period of time

Keeping track of the exercise history (Fig. 7 and Fig. 8) and other physiological data (Fig 9) will show the patients' progress over time and hopefully motivate a patient to continue with the exercises and lifestyle changes and get the benefits of complying with the cardiac rehabilitation programmes.

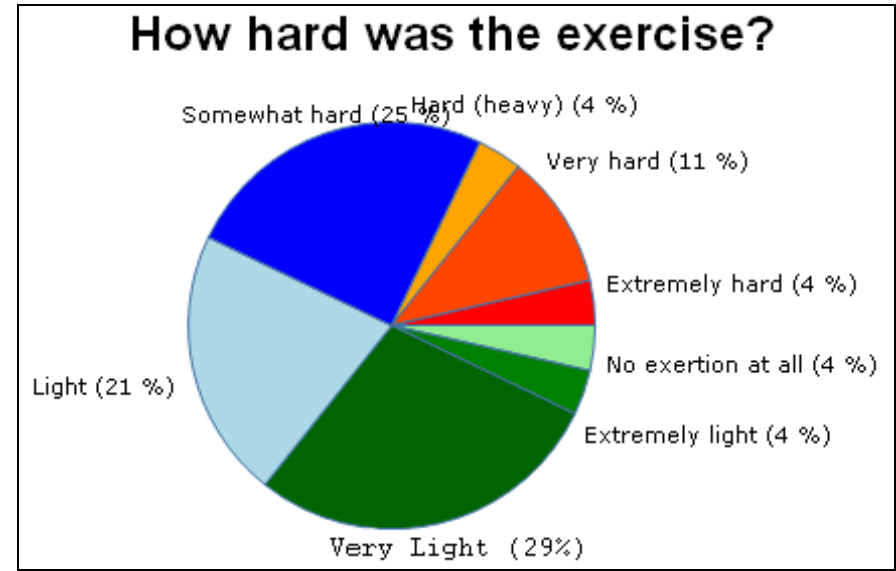

Figure 8: RPE History

Rehabilitation staff can view and comment on the physiological data of their patients and reports can be generated for review by other specialists or GPs.

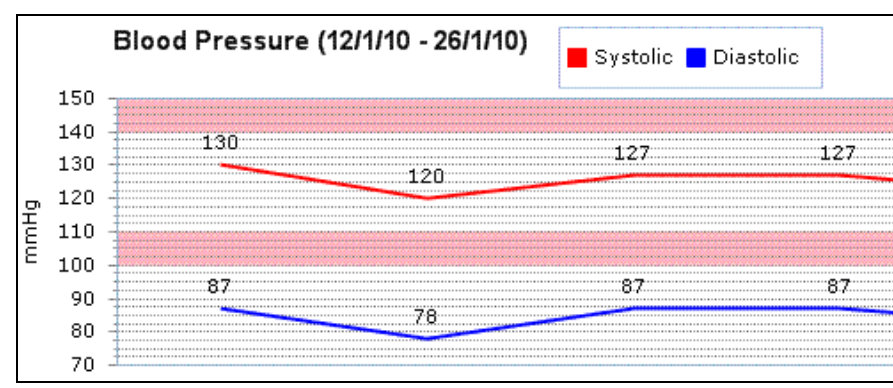

Figure 9: Blood Pressure History

\section{TRIAL WITH THE ROYAL NORTH SHORE HOSPITAL}

This section describes how the mobile cardiac rehabilitation application is trialed as part of the cardiac rehabilitation programme of the Royal North Shore Hospital in Sydney, Australia. Patients that recently experienced an acute cardiovascular event can join the rehabilitation programme to exercise under supervision and receive information on how to make lifestyle changes. We use the CONSORT [9] recommended structure for this section.

\section{A. Specific objectives}

The main objectives for this trial are to:

- Demonstrate that the outcomes of a cardiac rehabilitation programme can be improved using our novel mobile application.

- Study how our solution compares to conventional rehabilitation methods in terms of physical and psychological improvement and in helping the patient improving their vital signs (e.g. lower the bollod pressure) and making lifestyle changes.

- Obtain feedback from patients and health specialists regarding usability and practicability of the software and hardware used to monitor cardiac rehabilitation.

Secondary objectives are to: 
- Investigate whether the use of the novel application provides clinically meaningful reassurance to patients during their cardiac rehabilitation .

- Obtain feedback on the usefulness/efficiency of the application for cardiac patients using the personal health monitor application. (e.g. usefulness of daily reminders to measure weight/blood pressure, usefulness of the exercice videos).

- Obtain data on technical, clinical and psychological experiences.

Based on feedback of a previously conducted trial with over 90 patients with the Personal Health Monitor [12], we are confident that the user interface is usable by motivated patients of any age.

\section{B. Eligibility criteria for participants and the settings and} locations where the data are collected.

The patients are low risk patients and the following inclusion criteria will be applied:

- $\quad$ The patient has stable ischaemic heart disease - i.e. low immediate risk and ambulant.

- $\quad$ The patient is in a rehabilitation programme.

- The patient is willing and able (by language, motor skills education) to use new technology.

Exclusion criteria will be:

- Advanced or end stage heart disease.

- Other advanced and serious medical illness such as terminal cancer or major disability.

- An implanted pacemaker or defibrillator.

- Poor understanding of technical procedures by virtue of language, motor skill or education.

- Patients with occupational restrictions on the use of the technology (e.g. arc welders, airline employees).

The data is collected on the mobile phone for 24 patients and on paper for 12 patients. Data is not collected for the patients part of the reference group.

C. How sample size was determined and, when applicable, explanation of any interim analyses and stopping rules.

- $\quad$ The sample size is limited by the number of ECG, blood pressure and weight sensors that are available. At the time of writing we have 12 complete sets. After six months we will reiterate the trial with another group of 48 patients or larger group if more sensors are available.

\section{How participants are allocated to interventions}

An information leaflet about the novel mobile rehabilitation application is distributed during five consecutive rehabilitation sessions and a researcher is available during each session to discuss the trial with patients interested in participating.

A medical professional interviews and screens potential patients. High risk patients are excluded and 36 patients interested in trialing the new application are selected. Informed written consent is obtained from these patients.
The subjects are then randomly divided in three groups of twelve patients.

- The first group uses all the features of the mobile rehabilitation application consisting of the mobile phone and one or more sensors to monitor their physiological data.

- The second group uses the mobile rehabilitation application without sensors and they have to enter all data manually into the application.

- The third group does not use the mobile rehabilitation application and keep track of their progress and measurements on paper.

To ensure that the results are not influenced by paying extra attention to patients using the application, all 36 patients are contacted each month for the duration of the trial. In addition, 12 patients are randomly selected that are not interested in the trial. This group acts as a reference group. Table 1 summarizes the trial features for each group.

TABLE 1: TRIAL USING 4 PARALLEL GROUPS

\begin{tabular}{|l|c|c|c|c|}
\hline & $\begin{array}{c}\text { Group1 } \\
(12)\end{array}$ & $\begin{array}{c}\text { Group2 } \\
(12)\end{array}$ & $\begin{array}{c}\text { Group 3 } \\
\text { (12) }\end{array}$ & $\begin{array}{c}\text { Group 4 } \\
\text { (12) }\end{array}$ \\
\cline { 2 - 5 } & \multicolumn{3}{|c|}{ Motivated } & Others \\
\hline $\begin{array}{l}\text { Phone \& } \\
\text { PHM }\end{array}$ & $\checkmark$ & $\checkmark$ & $\begin{array}{c}\mathbf{x} \\
\text { paper log }\end{array}$ & $\mathbf{x}$ \\
\hline $\begin{array}{l}\text { Use Web } \\
\text { based } \\
\text { system }\end{array}$ & $\checkmark$ & $\checkmark$ & $\mathbf{x}$ & $\mathbf{x}$ \\
\hline $\begin{array}{l}\text { Wireless } \\
\text { sensors }\end{array}$ & $\checkmark$ & $\begin{array}{c}\mathbf{x} \\
\text { manual }\end{array}$ & $\begin{array}{c}\mathbf{x} \\
\text { manual }\end{array}$ & $\mathbf{x}$ \\
\hline $\begin{array}{l}\text { Monthly } \\
\text { email or } \\
\text { phone } \\
\text { contact }\end{array}$ & $\checkmark$ & $\checkmark$ & $\checkmark$ & $\mathbf{x}$ \\
\hline
\end{tabular}

E. Precise details of the interventions intended for each group and how and when they were actually administered.

On entering the programme, all patients are assessed on several factors such as

- Physical and psychosocial condition

- Symptom recognition

- Cardiac risk factors

- Past medical history

All patients perform a six minute walk test where their Rate of Perceived Exertion (RPE) is determined as well as oxygen saturation and heart rate. The results form the basis of their individualized exercise programme.

The physiotherapist advices the patients of group 1, 2 and 3 on the physiological data they should keep track of. For group 1 , he explains how to use the physiological sensors in conjunction with the mobile rehab application. For group 2 and 3 , no sensors are provided but it is up to each individual to check their physiological data using their own equipment (e.g. 
blood pressure monitor) or other methods (e.g. taking their pulse).

Group 1 and 2 patients attend a 20 minute tutorial on how to use the mobile cardiac rehabilitation application. A four page guide with screenshots is handed out that summarises the main features. The rehabilitation application is personalised based on the patient's profile and personal requirements (e.g. max heart rate for the exercises).

During the first six weeks of the rehabilitation program (inhouse period), all the patients discuss their progress with a nurse and a physiotherapist assists them in their cardiovascular and muscle strengthening exercises. Group 1 and 2 patients use the mobile rehabilitation application while doing their exercises. In addition, group 1 patients wear the ECG monitor during the sessions. This allows them to become familiar with the application while they are still being supervised. It also allows the physiotherapist to adjust threshold parameters for the exercises. Group 3 patients need to keep track of their progress on paper.

At the end of the six weeks programme, all patients are assessed and the 24 patients using the mobile rehabilitation application are assessed on the correct use of the application. They should have gained enough confidence to do the exercises without supervision of the hospital using the mobile rehabilitation application and to synchronize the data with the health centre server.

We keep in touch with group1, 2 and 3 patients with a monthly phone call. These calls have been introduced to make sure the 3 groups receive the same attention from the researchers. The calls are informal and used to check if there are any problems related to the trial. We only call people that did not contact us that month to try and balance the duration of the calls for each patient.

After 6 months all patients come to the rehabilitation centre for a reassessment. The health professionals have online access to all physiological and exercise data from group 1 and 2 and can easily assess their progress. The web based rehabilitation application supports them in preparing their report. The health professionals can also check the paper logs from group 3.

\section{F. Clearly defined outcome measures}

In order to get results as complete and reliable as possible, we use a mix of automatic and manual data collection over the 6 month period. On the mobile phone, the application collects the data coming from the sensors (or entered manually by the patient). The application keeps a log file of the date and the duration of its use and keeps track of the number of times a patient uses a particular feature (e.g. view exercise video).

On the back end, each time a person connects to the server (patient, technician or health professional), we record the date and the duration of the session and the features used during that session (e.g. view exercise summary, print ECG trace, create report).

At the rehabilitation centre, we collect data for the 48 patients at admission, during the 6 weeks in-house and during the reassessment after 6 months. The data collected includes the measurement of physical endurance using the 6 minute walk test[13], cholesterol, blood pressure and weight.

Over the 6 months trial period, the 36 participants record all their contacts with health services, including the number and purpose of visits to their family doctor (GP), medical specialist, hospital, nurse, pharmacist, community health centre. We also keep track how often a patient contacts us and the reason for the call. This is used to calculate the cost effectiveness of remote monitoring.

After the re-assessment, we ask the 36 patients and the health professionals involved in the study to fill in a survey. Information collected are:

- General information (age, level of education, profession, computer literacy

- $\quad$ The main reasons why they volunteered to use the mobile rehabilitation application (e.g.: peace of mind, wanting to feel in charge of their health condition).

- An overall rating of the usability of the novel application and a rating of the usefulness for the different functionalities.

- Whether they benefited from using the application (e.g. anxiety level, peace of mind, quality of life).

- A rating of the usefulness of the application for other health related aspects (e.g. continuous heart monitoring, diabetes monitoring).

- The main advantages and disadvantages of using the mobile rehabilitation application and whether they would recommend the application to others in a similar situation.

- $\quad$ The money they are willing to spend on the application by either renting or buying it.

After the trial, the data collected over the 6 month period will be used to compare the 4 groups and obtain answers to the following questions:-

1. Is cardiac rehabilitation feasible using our novel application?

2. How does it compare to conventional rehabilitation methods in terms of physical and psychological improvement and in helping the patient improving their vital signs and making lifestyle changes?

3. Does the use of the novel application provide clinically meaningful reassurance to patients during their cardiac rehabilitation .

We will obtain feedback from patients and health specialists regarding usability and practicability of the software and hardware used and collect data on technical, clinical and psychological experiences. 


\section{CONCLUSIONS}

The novel mobile rehabilitation application described in this paper aims at encouraging patients to do their exercises and improve their confidence by constant local monitoring. It can aid patients to achieve the required life style changes by monitoring their process but also to remind them to do their exercises.

This paper presents the set up of the trial which will commence in August 2010. The value and significance of this research comes from assessing how economical nonproprietary technology can enhance clinical management. This will extend present technologies for personal remote monitoring and training methods.

Our application can be used by rehabilitation clinics (inhouse) but also by outpatients that have not access to rehabilitation services or wish to continue after the in-house sessions. It does not replace rehabilitation clinics since human supervision and personal health checks are essential to the rehabilitation process. It complements those services by recording the patient's physiological data and activities. It offers rehabilitation centres to remotely evaluate their patient's health and progress. We believe that this application can help cardiac patients making long term lifestyle changes and complying with a rehabilitation programme.

\section{ACKNOWLEDGMENT}

The Personal Health Monitor is supported by the University of Technology, Sydney and is currently under commercial development.

\section{REFERENCES}

[1] American Heart Association. Cardiac Rehabilitation. http://www.americanheart.org/presenter.jhtml?identifier=3047638

[2] University of Alberta, 'Remote Monitoring Saves Lives Of Heart Patients, Research Shows', ScienceDaily April 2007.

[3] Thompson, D. R., Bowman, G. S., Kitson, A.L., Bono, D. P. de, Hopkins, A., 'Cardiac rehabilitation in the United Kingdom: guidelines and audit standards'. Heart. Vol. 75. pp. 89-93, 1996.

[4] Horgan, J., Bethel, H., Carson, P., Davidson, C., Julian, D., Mayou, R. A., Bagle, R. Working party report on cardiac rehabilitation. British Heart Journal. Vol 67. pp. 412-418, 1992.

[5] Thomson, D. R., Bono, D. P. 'How valuable is cardiac rehabilitation and who should get it?’, Heart. Vol 82. pp. 545-546, 1999.

[6] Normand S-L. T., Ades P., and Prottas J., Rehabilitation significantly underused after heart attack and bypass surgery, Circulation: Journal of the American Heart Association, 25-Sep-2007.

[7] Cardiomobile -Remote Monitoring System, http://www.ihbi.qut.com/research/injury/cardiomobile/index, June 2008.

[8] Bidargaddi N.P, Sarela A., 'Activity and heart rate-based measures for outpatient cardiac rehabilitation', Methods of Information in Medicine; 47(3):208-216, 2008.

[9] Moher D, Schulz KF, Altman DG. 'The CONSORT statement: revised recommendations for improving the quality of reports of parallel-group randomised trials’357(9263):1191-1194, Lancet 2001.

[10] Gay V., Leijdekkers P., 'A Health Monitoring System Using Smart Phones and Wearable Sensors', Special Issue on 'Smart Sensors in Smart Homes' International Journal of Assistive Robotics and Mechatronics, Vol. 8, No. 2, 2007

[11] Personal Health Monitor, http://www.personalhealthmonitor.net .

[12] Gay V., Leijdekkers P. and Barin E., 'Trial Results of a Novel Cardiac Rhythm Management System using Smart Phones and wireless ECG Sensors', ICOST 2009, France, July 2009.

[13] American Thoracic Society, 'Guidelines for the six-minute walk test'. Am J Respir Crit Care Med, 166:111-117, 2002. 